\begin{tabular}{|c|c|}
\hline Title & Robust Optimization Considering Probabilistic Magnetic Degradation \\
\hline Author(s) & Hidaka, Y uki; Furui, Shintaro; Igarashi, Hajime \\
\hline Citation & $\begin{array}{l}\text { IEEE transactions on magnetics, 51(3), } 7401204 \\
\text { https://doi.org/10.1109/T MA G.2014.2353653 }\end{array}$ \\
\hline Issue Date & 2015-03 \\
\hline Doc URL & http:/hdl.handle.net/2115/59619 \\
\hline Rights & $\begin{array}{l}\text { (c) } 2015 \text { IEEE. Personal use of this material is permitted. Permission from IEEE must be obtained for all other uses, in } \\
\text { any current or future media, including reprinting/republishing this material for advertising or promotional purposes, } \\
\text { creating new collective works, for resale or redistribution to servers or lists, or reuse of any copyrighted component of } \\
\text { this work in other works. }\end{array}$ \\
\hline Tyре & article (author version) \\
\hline File Information & 70027.pdf \\
\hline
\end{tabular}

Instructions for use 


\title{
Robust Optimization Considering Probabilistic Magnetic Degradation
}

\author{
Yuki Hidaka ${ }^{1}$, Shintaro Furui ${ }^{1}$ and Hajime Igarashi ${ }^{1}$, Member IEEE \\ ${ }^{1}$ Graduate School of Information Science and Technology, Hokkaido University, \\ Kita 14, Nishi 9, Kita-ku, Sapporo, 060-0814, JAPAN
}

\begin{abstract}
This paper presents robust topology optimization of electromagnetic machines taking account of magnetic degradation caused by mechanical and thermal stresses in punching, shrinking fitting and other manufacturing processes. The topology optimization is performed using two methods; one is the robust genetic algorithm in which random noises are added to the magnetic-characteristic parameters, and another takes the deviations in the objective and constraint functions due to the degradation into account. These methods are applied to optimization of the flux barrier shapes in an IPM motor to find that one can successfully realize robust design.
\end{abstract}

Index Terms — Finite element method, robust optimization, topology optimization, magnetic degradation.

\section{INTRODUCTION}

I $t$ has been pointed out that there can exist significant $\mathbf{I}_{\text {magnetic degradation in the magnetic materials used in }}$ electric machines [1-3]. They are attributed to thermal and kinetic stresses in punching, shrinking fitting and other manufacturing processes. The magnetic degradation leads to deterioration in machine performance and efficiency. For this reason, efforts have been paid to measure and analyze the magnetic degradation to keep the performance of finished machines within the specification [4-5]. We need design optimization methods taking possible magnetic degradation into account to make further improvement of machine performance.

There have been several approaches to consider possible changes in material properties and machine shapes for design optimization. For example, sensitivity in the objective and constraint functions is evaluated by sampling their values at the neighborhood lattice of the current point in the parameter space [6]. This method is, however, computationally prohibitive for population based optimization methods including genetic algorithm (GA). The robust design method based on deterministic multi-objective optimization [7] and Taguchi method [8] do not have sufficient searching ability in high-dimensional design space.

The robust GA using artificial noise [9], which will be call RGA hereafter, has no increase in computational burden in comparison with the conventional non-robust optimization method. Moreover, RGA has been shown to provide solutions to high-dimensional problems which are robust for parameter changes. Furthermore, we have yet another effective robust optimization method in which the sensitivity is evaluated from the adjoint variable method (AVM) without sampling [10]. It has been unclear if the last two methods are effective for optimization considering possible magnetic degradation. Moreover, validity of these methods for topology optimization, in which material shapes are flexibly changed without design parameters, also remains unclear. In this paper, we will discuss if they are useful for topology optimization of electric

Manuscript received January 1, 2014. Corresponding author: Hajime Igarashi (e-mail: igarashi@ssi.ist.hokudai.ac.jp).

Digital Object Identifier inserted by IEEE machines which have possible magnetic degradations. In particular, we focus on the on/off topology optimization performed by GA [11]. We compare the performance of usual GA with two robust methods, RGA and AVM-based GA (AVM-GA) in the optimization of the rotor shape of an interior permanent magnetic (IPM) motor.

\section{OPTIMIZATION METHOD}

Let us consider here a magnetostatic problem although the present method can be applied to quasi-static and wave problems. The two-dimensional magnetostatic field on the $x-y$ plane is governed by

$\nabla \cdot(\nu \nabla A)=-J-(\operatorname{rot} \boldsymbol{M})_{Z}$,

where $v, A, J$ and $\boldsymbol{M}$ denote magnetic reluctivity, the $z$ components of vector potential and current density, and magnetization vector of permanent magnets, respectively. To solve (1) using finite element method (FEM), the unknown vector potential $A$ is discretized as follows:

$A=\sum_{i=1}^{n} N_{i} A_{i}$,

where $N_{i}$ is the scalar interpolation function, $n$ is the number of nodal points. By applying the Galerkin method to (1), we obtain the FE equation

$\sum_{j} A_{j} \int_{\Omega} v \nabla N_{j} \cdot \nabla N_{i} \mathrm{~d} S=\int_{\Omega} N_{i} J \mathrm{~d} S+\int_{\Omega} N_{i}(\operatorname{rot} \boldsymbol{M})_{z} d S$,

for $i=1,2, \ldots, n$. Eqn. (3) is expressed in the matrix form $K \boldsymbol{a}=\boldsymbol{b}$, where $K, \boldsymbol{a}$ and $\boldsymbol{b}$ denote the FE matrix which is symmetric and sparse, unknown and source vectors, respectively. In the optimization process, Eqn. (3) is repeatedly solved to evaluate the objective and constraints functions.

\section{A. Parameterization of $\mathrm{BH}$ curves}

In the present method, considering possible magnetic degradation, the $\mathrm{BH}$ characteristic is modeled by

$$
v(H)=\frac{H}{B}=\frac{H}{k_{1}\left[1-\exp \left(-k_{2} H\right)\right]+\mu_{0} H}
$$


where $H$ and $B$ are magnetic field and induction. Moreover $k_{1}$ and $k_{2}$ are constants which have uncertainties. Figure 1 shows the dependence of the $\mathrm{BH}$ curves on $k_{1}$ and $k_{2}$. Other parameterization of the $\mathrm{BH}$ curves can be employed for the following robust optimization method.

In the nonlinear FEM, differentiation of the magnetic reluctivity is computed by

$\frac{\partial v}{\partial A}=\frac{\partial v}{\partial H}\left(\frac{\mathrm{d} B}{\mathrm{~d} H}\right)^{-1} \frac{\partial B}{\partial A}$

\section{B. Adjoint variable method aided GA (AVM-GA)}

In this method, the sensitivities in objective and constraint functions are evaluated to consider their deviation during the optimization process. The sensitivity of objective function $F$ is computed from

$\frac{\mathrm{d} F}{\mathrm{~d} k_{i}}=\frac{\partial F}{\partial k_{i}}+\left(\frac{\partial F}{\partial \boldsymbol{a}}\right)^{\mathrm{t}} \frac{\partial \boldsymbol{a}}{\partial k_{i}}$.

In Eqn. (6), it may be difficult to compute $\partial \boldsymbol{a} / \partial k_{i}$ analytically. The adjoint variable method is thus employed in which the augmented objective function defined by

$$
\hat{F}=F+\mathbf{z}^{\mathrm{t}}(\mathrm{K} \boldsymbol{a}-\boldsymbol{b}),
$$

is considered where $\mathbf{z}$ is an adjoint variable. Note that $\hat{F} \approx F$ assuming that $\boldsymbol{a}$ satisfies the FE equation in good accuracy. Differentiation of Eqn. (7) with respect to $k_{i}$ leads to

$$
\frac{\mathrm{d} \hat{F}}{\mathrm{~d} k_{i}}=\frac{\partial F}{\partial k_{i}}+\mathbf{z}^{\mathrm{t}} \frac{\partial \mathrm{K}}{\partial k_{i}} \boldsymbol{a}+\left[\mathbf{z}^{\mathrm{t}} \mathrm{K}+\left(\frac{\partial F}{\partial \boldsymbol{a}}\right)^{\mathrm{t}}\right] \frac{\partial \boldsymbol{\alpha}}{\partial k_{i}} .
$$

We assume that $\mathbf{z}$ satisfies

$$
\mathrm{K} \mathbf{z}=-\frac{\partial F}{\partial \boldsymbol{a}} \text {. }
$$

Consequently, the sensitivity of $F$ can be evaluated from

$\frac{\mathrm{d} F}{\mathrm{~d} k_{i}} \approx \frac{\partial F}{\partial k_{i}}+\mathbf{z}^{\mathrm{t}} \frac{\partial \mathrm{K}}{\partial k_{i}} \boldsymbol{a}$.

The standard deviation $\sigma_{F}$ of $F$ is computed from

$\sigma_{F}=\sqrt{\sum_{i}\left(\frac{\mathrm{d} F}{\mathrm{~d} k_{i}}\right)^{2} \sigma_{i}^{2}}$,

where $k_{i}$ is assumed to be linearly independent and $\sigma_{i}$ denotes the standard deviation of $k_{i}$.
To take robustness into account, the optimization problem, $F \rightarrow$ min., is modified to

$F+\alpha \sigma_{F} \rightarrow \min$. ,

where $\alpha$ is an arbitrary constant. The robustness of the final solution would be improved while its fitness would be deteriorated if we increase $\alpha$, and vice versa. For maximizing nrnhlome waro rnncider $F-\alpha \sigma_{F} \rightarrow$ max. instead of (12). On nstraint $G<0$ is modified to

Jdified to $G-\beta \sigma_{G}>0$.

Algorithm (RGA)

der the probabilistic effect of magnetic 1 noise $\boldsymbol{\delta}$ is introduced to the material TS:

$t$ of the RGA process is the same as the 0000 :re is small differences in evaluation and sefectuortainties pross. In GA, elite selection is often used to improve the convergence. The usual elite selection is not used in RGA because the fitness of the individual is fluctuated by the effect of noises. Procedure of the RGA is summarized below where $F(\boldsymbol{x}, \boldsymbol{\delta})$ denotes the fitness of an individual $\boldsymbol{x}$ to whose material parameters noise $\boldsymbol{\delta}$ is introduced.

1) Initial population at $t=1$ is generated randomly.

2) The elite $\boldsymbol{x}$ which has the highest fitness $F(\boldsymbol{x}, \mathbf{0})$ at generation $t$ is selected.

3) The elite candidate $\boldsymbol{y}$ which has the highest fitness $F(\boldsymbol{y}, \mathbf{0})$ at generation $t+1$ is selected.

4) Fitness $F(\boldsymbol{x}, \boldsymbol{\delta})$ is compared with $F(\boldsymbol{y}, \mathbf{0})$. If $F(\boldsymbol{y}, \mathbf{0})$ is better than $F(\boldsymbol{x}, \boldsymbol{\delta}), \boldsymbol{x}$ is replaced by $\boldsymbol{y}$ as the new elite.

5) Steps 3-5 are repeated until the iteration number reaches the maximum.

In the above procedure, the fitness of each individual is evaluated using FEM.

\section{OPTIMIZATION RESULTS}

\section{A. Simple Test Model}

Before we consider the optimization of motors, we test basic performances of GA, AVM-GA and RGA applied to the following toy optimization problem:

$\begin{aligned} F(x)= & C_{1} e^{-\frac{\left(x-x_{1}\right)^{2}}{2 \sigma_{1}^{2}}}+C_{2} e^{-\frac{\left(x-x_{2}\right)^{2}}{2 \sigma_{2}^{2}}} \rightarrow \text { max., } \\ & \text { sub. to } G(x)=x-x_{2}<0,\end{aligned}$

where the parameters are set as follows: $C_{1}=2.0, C_{2}=$ $1.0, x_{1}=0.5, x_{2}=4.0, \sigma_{1}=0.1, \sigma_{2}=1.5$. To consider the constraint, we modify the original problem to $F^{\prime}=F-$ $u(G) \rightarrow$ max., where $u$ is the step function. Note that there is a sharp drop in $F^{\prime}$ for $x \geq x_{2}$. It is assumed that coordinate $x$ corresponds to the material parameter; uniform noise $-0.3 \leq$ $\delta \leq 0.3$ is added to $x$ in RGA. We set $\sigma=0.6 / \sqrt{12}, \alpha=$ $2, \beta=1$ in (12) and (13) for AVM-GA. We carry out 20 runs for each method. The results are shown in Fig.2. We find that 
AVM-GA and RGA can provide robust solutions at which $F^{\prime}$ is insensitive for possible changes in $x$.

\section{B. Optimization model of IPM motor}

The present method is applied to the IPM-motor model

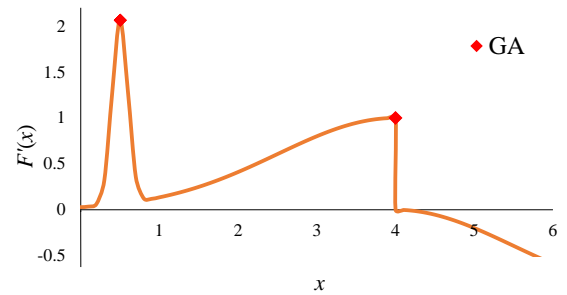

(a) GA. 3 and 17 solutions exist near $x_{1}$ and $x_{2}$, respectively.

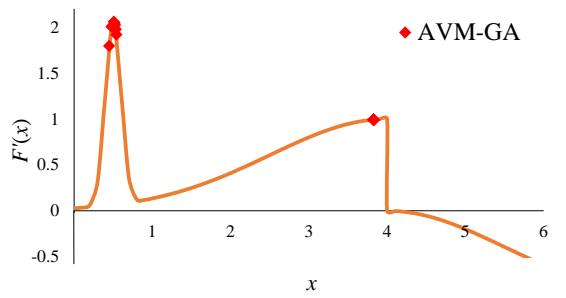

(b) AVM-GA. 7 and 13 solutions exist near $x_{1}$ and $x_{2}$,

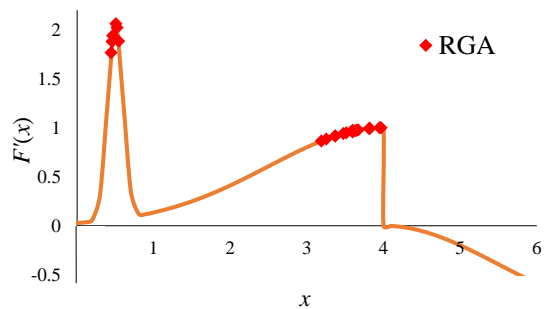

(c) RGA. 6 and 14 solutions exist near $x_{1}$ and $x_{2}$, respectively.

Fig. 2 Optimization results for toy problem

shown in Fig. 3. Due to the symmetry, 1/4 region of the model is analyzed. The purpose of this optimization is to minimize torque ripple under the constraint that the torque average is larger than the reference value. The optimization problem is defined by

$$
F=\frac{T_{\max }-T_{\min }}{T_{\text {avg }}} \rightarrow \min .,
$$

sub. to $G=T_{r e f}-T_{a v g}<0$,

where $T_{\max }, T_{\min }$ denote the maximum and minimum torques and $T_{\text {avg }}$ represents the torque average, and we assume that $T_{\text {ref }}=4.0 \mathrm{Nm}$. In Table I, the analysis conditions and motor specification are summarized. The problem is modified to $F^{\prime}=F+1000 u(G) \rightarrow$ min. in the optimization. The parameters $\boldsymbol{k}=\left(k_{1}, k_{2}\right)$ are assumed to have uncertainty which obeys the exponential probability density functions $\exp \left(\lambda_{i} k_{i}\right)$ where $k_{1} \leq 2.15, k_{2} \leq 1 / 4300$ and $\lambda_{1}=35, \lambda_{2}=$ $9.2 \times 10^{4}$. The reason why we assume the asymmetric distribution is that we consider degradation in the magnetic properties. The corresponding noise is generated in RGA, and we assume $\alpha=\beta=0.4$ in AVM-GA. After the optimization, robustness of the solutions is evaluated using the Monte Carlo method in which the deviation of the objective function $\sigma_{F}$ and the constraint violation probability $P_{G}$ are computed.

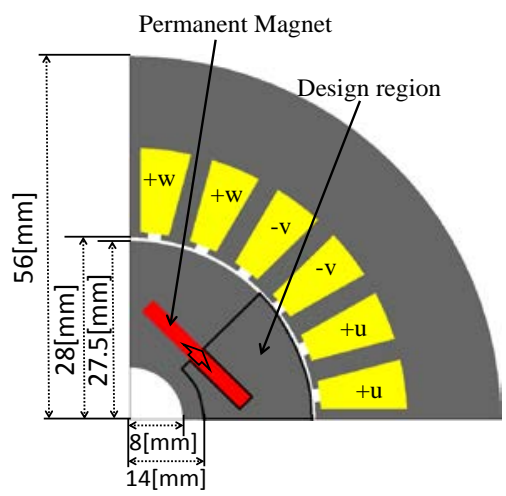

Fig. 3. IPM motor for optimization

TABLE I

ANALYSIS CONDITIONS AND SPECIFICATIONS

\begin{tabular}{cc}
\hline \hline Rotation speed (rpm) & 3000 \\
Armature current (A·turn) & 420 \\
Phase of current (degree) & 20 \\
Residual flux density of PM (T) & 1.0 \\
Width of teeth (mm) & 3.3 \\
Length of Coil (mm) & 25.9 \\
Thickness of PM (mm) & 2.5 \\
Width of PM (mm) & 21 \\
\hline \hline
\end{tabular}

\section{Optimization Results}

The resultant shapes obtained by conventional GA, AVMGA and RGA are shown in Fig. 4. They are the best solutions in 20 runs for each method. We can see that the feature of the rotor shapes obtained by GA and AVM-GA are similar; there are two side and one central flux barriers. On the other hand, the result of RGA has no central flux barrier.

Optimization results are summarized in Table II. The solution obtained by RGA has clearly smaller value of $\sigma_{F}$ in comparison with those obtained by GA and AVM-GA. Moreover, the constraint violation probabilities $P_{G}$ of the solutions obtained by RGA and AVM-GA are clearly smaller than that of GA. It is remarkable that the solution of GA always violates the constraint even for small perturbation in $\boldsymbol{k}$. On the other hand, the solutions obtained by AVM-GA and RGA have sufficient margins for $G$. For this reason, these solutions are robust against the fluctuation of magnetic characteristic. The torque waves of all solutions are shown in Fig. 5.

Fig. 6 shows the frequency distributions of $F$ and $G$ which are computed by the Monte Carlo method. It is found that the distribution of $F$ for RGA is clearly narrower than two other distributions. On the other hand, the central values of $F$ for RGA and AVM-GA are not expected to be smaller than that of GA from their principles. Thus, the difference in the central values of $F$ in Fig. 6(a), which receives stochastic effect, has no significant meaning. In Fig 6(b), one can see that the values of $G$ for RGA and AVM-GA distribute almost in the safe side whereas they violate the constraint for GA.

\section{CONCLUSION}

In this paper, we have presented the robust optimization 


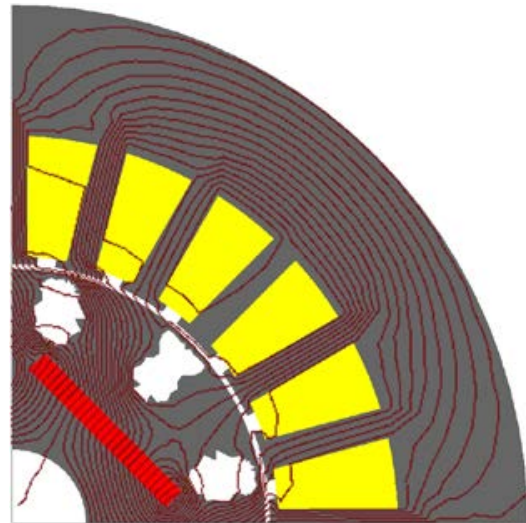

(a) GA

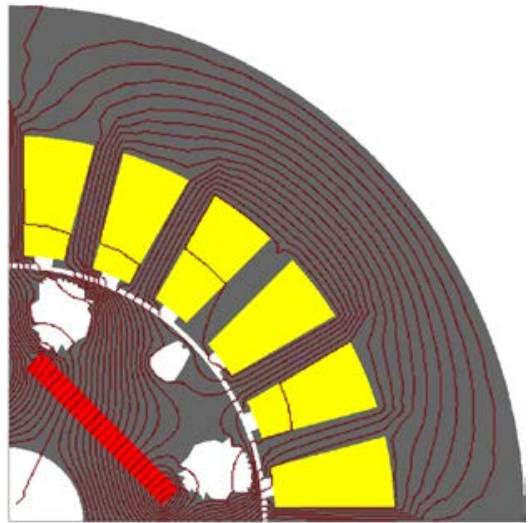

(b) AVM-GA

Fig. 4. Optimized shapes with flux distribution.

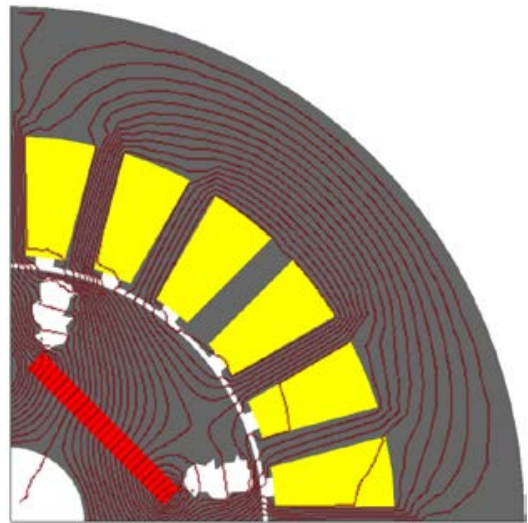

(c) RGA methods considering magnetic degradation. The BH curves are expressed by the explicit function which has uncertainties in parameters.

The present methods, RGA and AVM-GA, have been shown to provide the optimized rotor shapes of the IPM motor which has robustness in the constraint. Moreover, it has been shown that the solution of RGA also has robustness in the objective function.

TABLE II

OPTIMIZATION RESULTS OF IPM MOTOR

\begin{tabular}{cccc}
\hline & \multicolumn{4}{c}{ OPTIMIZATION RESULTS OF IPM MOTOR } \\
mean $F, G$ & $0.235,-0.0718$ & $0.280,-0.313$ & $0.179,-0.432$ \\
$\sigma_{F}\left(\mathrm{x} 10^{-3}\right)$ & 4.1 & 3.7 & 1.9 \\
$P_{\mathrm{G}}$ & 1.0 & 0.039 & 0.010 \\
\hline \hline
\end{tabular}

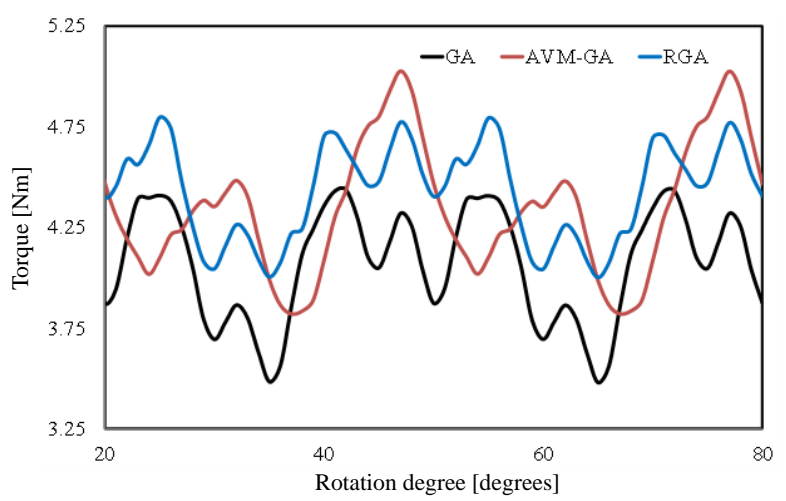

Fig. 5. Comparison of Torques

\section{REFERENCES}

[1] A. Schoppa, J. Schneider and C. -D. Wuppermann, "Influence of the manufacturing process on the magnetic properties of non-oriented electrical steels," Journal of Magnetism and Magnetic Materials, vols. 215-216, pp. 74-78, 2000.

[2] E. G. Araujo, J. Schneider, K. Verbeken, G. Pasquarella and Y. Houbaert, "Dimensional effects on magnetic properties of Fe-Si steels due to laserand mechanical cutting,” IEEE Trans. Magn., vol. 46, no. 2, pp. 213-216, 2010.

[3] Y. Kurosaki, H. Mogi, H. Fujii, T. Kubota and M. Shiozaki, "Importance of punching and workability in non-oriented electrical steel sheets,” J. Magn. Mag. Mat., vol. 320, no. 20, pp. 2474-2480, 2008.

[4] A.J. Clerc and A. Muetze, "Measurement of Stator Core Magnetic Degradation during the manufacturing process," IEEE Trans. Magn., vol. 48, no. 4, pp. 1344-1352, 2012.

[5] M. Bali, H. De Gersem and A. Muetze, "Finite-element modeling of magnetic material degradation due to punching," IEEE Trans. Magn., vol. 50, no. 2, 7018404, 2014.

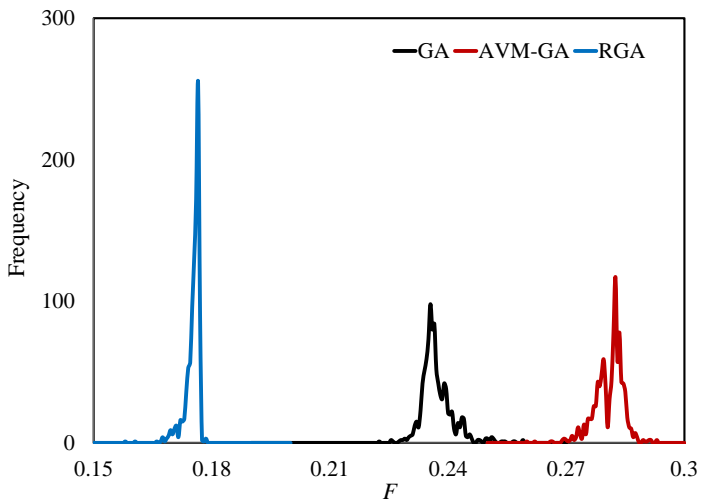

(a) Objective function $F$.

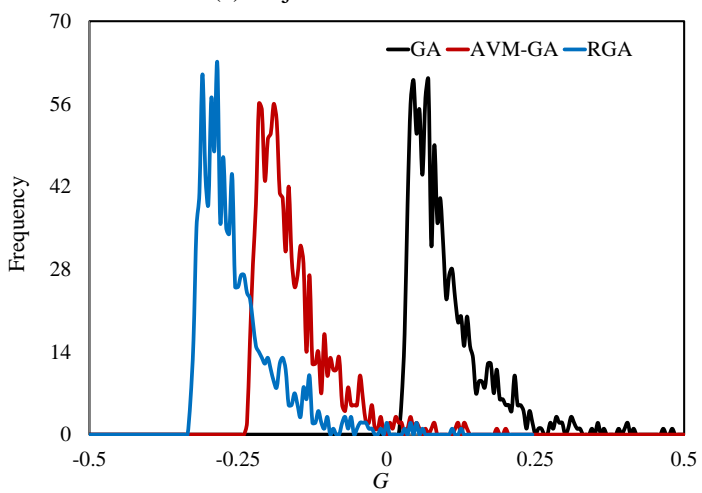

(b) Constraint function $G$

Fig.6 . Frequency distributions computed by Monte Carlo method.

[6] G. Steiner, A. Weber and C. Magele, "Managing uncertainties in electromagnetic design problems with robust optimization," IEEE Trans. Magn., vol. 40, no. 2, pp. 1094-1099, 2004.

[7] S-B. Yoon, I-S. Jung, D-S. Hyun, "Robust shape optimization of electromechanical devices," IEEE Trans. Magn., vol. 35, no. 3, pp. 1710-1713, 1999.

[8] H.T. Wang, Z.J. Liu, S.X. Chen, J.P. Yang, "Application of Taguchi method to robust design of BLDC motor performance, " IEEE Trans. Magn., vol. 35, no. 5, pp. 3700-3702, 1999.

[9] T. Maruyama and H. Igarashi, "An effective robust optimization based on genetic algorithm,” IEEE Trans. Magn., vol. 44, no. 6, pp. 990-993, 2008.

[10] T. Maruyama, K. Watanabe and H. Igarashi, "A robust optimization based on adjoint variable method," COMPEL, vol. 29, no. 9, pp. 15241532, 2010.

[11] Y. Hidaka, T. Sato, H. Igarashi, "Topology optimization method based on on-off method and level-set approach,” IEEE Trans. Magn., vol. 50, no. 2, 7015204, 2014.

[12] Technical report of the institute of electrical engineering of Japan, industry application society, no. 776, 2000. 